\title{
Proton Pump Inhibitors Did Not Increase Risk of Pneumonia in Patients With Chronic Obstructive Pulmonary Disease
}

\author{
Shou-Wu Lee, b, e, Ching-Heng Lin ${ }^{c}$, Han-Chung Lien ${ }^{\mathrm{a}, \mathrm{d}}$, Teng-Yu Lee ${ }^{\mathrm{a}, \mathrm{b}}$, Hong-Zen Yeh ${ }^{\mathrm{a}, \mathrm{c}}$, \\ Chi-Sen Chang ${ }^{\mathrm{a}, \mathrm{b}}$
}

\begin{abstract}
Background: Chronic obstructive pulmonary disease (COPD) involves the airways and pneumonia is a major cause of mortality. Proton pump inhibitors (PPIs) were found to have a positive association with pneumonia. The aim of this study was to investigate the impact of PPIs on the risk of pneumonia in patients with COPD.

Methods: This was a nationwide, population-based, case-control study using data from the National Health Insurance Research Database in Taiwan. The enrolled cases were defined as patients with COPD and appearance of pneumonia between 2001 and 2005. The control group was age- and sex-matched 1:2 with the cases without pneumonia. Potential confounders such as coronary artery disease, hypertension, diabetes mellitus, heart failure, chronic kidney disease, and prescriptions of glucocorticoids over 2 weeks, were included in the analysis. Prescriptions for PPIs were identified and entered into the analysis.
\end{abstract}

Results: A total of 10,131 COPD patients, including 3,377 cases with pneumonia and 6,754 without, were identified. There were 213 (5.3\%) and $436(6.5 \%)$ cases with concurrent PPIs in the two groups, respectively, and the risk of pneumonia was similar $(\mathrm{aOR}=0.96 ; 95 \%$ CI: 0.83 - 1.10). Further subgroup analysis found no differences for younger patients (younger than 70 years old; $a \mathrm{OR}=1.04 ; 95 \% \mathrm{CI}$ : $0.83-1.10$ ), elderly patients (older than 70 years old; $\mathrm{aOR}=0.96$; 95\% CI: $0.81-1.15$ ), short-term use of PPIs (less than 30 days; aOR $=1.12$; CI: 0.53 - 2.34), medium-term use of PPIs (30 - 90 days; aOR

Manuscript accepted for publication September 16, 2015

aDivision of Gastroenterology, Department of Internal Medicine, Taichung Veterans General Hospital, Taichung, Taiwan

bDepartment of Internal Medicine, Chung Shan Medical University, Taichung, Taiwan

'Department of Medical Research, Taichung Veterans General Hospital, Taichung, Taiwan

dDepartment of Internal Medicine, National Yang-Ming University School of Medicine, Taipei, Taiwan

${ }^{\mathrm{e} C}$ Corresponding Author: Shou-Wu Lee, Division of Gastroenterology, Department of Internal Medicine, Taichung Veterans General Hospital, Taichung, 1650 Taiwan Boulevard, Sec. 4, Taichung 40705, Taiwan.

Email: ericest@vghtc.gov.tw

doi: http://dx.doi.org/10.14740/jocmr2322w
$=0.86$; CI: $0.72-1.03$ ), or long-term use of PPIs (longer than 90 days; $\mathrm{aOR}=0.97$; CI: $0.81-1.15$ ).

Conclusion: PPIs did not contribute to a greater occurrence of pneumonia in COPD patients compared with non-users in this populationbased case-control study. Further research is required.

Keywords: Chronic obstructive pulmonary disease; Pneumonia; PPI

\section{Introduction}

Chronic obstructive pulmonary disease (COPD) involves the airways and is characterized by airflow limitation. Typical symptoms of COPD are dyspnea, chronic cough, and sputum production, and less common symptoms include wheezing and chest tightness $[1,2]$. Pneumonia is a major cause of death in COPD patients, with mortality rates as high as $11 \%$ [3]. Therefore, preventing pneumonia is vital to reducing morbidity and mortality in COPD patients.

Proton pump inhibitors (PPIs), introduced into clinical practice in 1988, became the mainstay of therapy for many acid-related gastrointestinal disorders [4]. Previous observational studies found a positive association between PPI use and community-acquired pneumonia [5-8]. However, the relationship between PPIs and pneumonia in patients with COPD has not been determined. The aim of this study was to provide formal evidence of the impact of PPIs on the risk of pneumonia in individuals with COPD.

\section{Patients and Methods}

This was a nationwide, population-based, case-control study. Claims data were obtained from the National Health Insurance Research Database (NHIRD) in Taiwan. The NHI provides coverage for over $98 \%$ of the island's population of approximately 23 million. The enrolled cases in this study were defined as patients with COPD (International Classification of Diseases, ninth revision, clinical modification code 493) between 2000 and 2005, and age older than 30 years. A diagnosis of pneumonia (ICD-9-CM codes 480 to 486) in the enrolled cases during this period was recorded. 
Table 1. Number of Baseline Characteristics in Newly Identified COPD Patients in $2000-2005$

\begin{tabular}{|c|c|c|c|}
\hline \multirow{2}{*}{ Variables } & \multicolumn{2}{|c|}{ Pneumonia } & \multirow{2}{*}{ P-value } \\
\hline & No $(n=6,754), n(\%)$ & Yes $(n=3,377), n(\%)$ & \\
\hline Age (years) & & & 1.000 \\
\hline Mean \pm SD & $68.3 \pm 12.6$ & $71.1 \pm 13.9$ & \\
\hline $30-39$ & $212(3.1)$ & $106(3.1)$ & \\
\hline $40-49$ & $400(5.9)$ & $200(5.9)$ & \\
\hline $50-59$ & $818(12.1)$ & 409 (12.1) & \\
\hline $60-69$ & $1,182(17.5)$ & $591(17.5)$ & \\
\hline$\geq 70$ & $4,142(61.3)$ & $2,071(61.3)$ & \\
\hline Sex & & & 1.000 \\
\hline Women & $2,204(32.6)$ & $1,102(32.6)$ & \\
\hline Men & $4,550(67.4)$ & $2,275(67.4)$ & \\
\hline \multicolumn{4}{|l|}{ Comorbidities } \\
\hline Hypertension & 3,237 (47.9) & $1,827(54.1)$ & $<0.001$ \\
\hline DM & $1,351(20.0)$ & $846(25.1)$ & $<0.001$ \\
\hline $\mathrm{HF}$ & $591(8.8)$ & $398(11.8)$ & $<0.001$ \\
\hline CAD & $1,816(26.9)$ & $1,009(29.9)$ & 0.002 \\
\hline CKD & $218(3.2)$ & $151(4.5)$ & 0.002 \\
\hline Glucocorticoids & $1,686(25.0)$ & $1,087(32.2)$ & $<0.001$ \\
\hline Drug use & & & 1.000 \\
\hline PPI & $436(6.5)$ & $213(6.3)$ & \\
\hline
\end{tabular}

tChi-square test. CAD: coronary artery disease; CKD: chronic kidney disease; COPD: chronic obstructive pulmonary disease; $\mathrm{DM}$ : diabetes mellitus; mean $\pm \mathrm{SD}$ : mean \pm standard derivation; $\mathrm{n}$ : numbers; PPI: proton pump inhibitor.

A total of 3,377 (33.3\%) individuals with pneumonia were identified during the period 2000 - 2005, and they were then age- and sex-matched 1:2 with 6,754 cases without pneumonia who served as the control group.

The following potential confounders were entered into the analysis, as follows: coronary artery disease (CAD) (ICD-9-CM codes: 410 to 414), hypertension (ICD-9-CM codes 401 to 405), diabetes mellitus (DM) (ICD-9-CM codes 250), heart failure (HF) (ICD-9-CM codes 428.00), and chronic kidney disease (CKD) (ICD-9-CM codes 585, 586, 588.8, 588.9, 250.4, 274.1, 403, 404, 404 and 440.1). Concurrent prescriptions of glucocorticoids over 2 weeks that could potentially confound the association between PPI use and pneumonia were also identified. The data files contained information on patients' prescriptions for PPIs, including

Table 2. Odds Ratios and $95 \%$ Confidence Interval of Pneumonia Associated With Risk Factors in Conditional Multivariate Logistic Regression Analysis

\begin{tabular}{lll}
\hline Drug use & OR $(\mathbf{9 5 \%}$ CI) & OR $^{\dagger}(\mathbf{9 5 \%}$ CI $)$ \\
\hline None & 1.00 (Reference) & 1.00 (Reference) \\
PPI & $1.00(0.87-1.15)$ & $0.96(0.83-1.10)$ \\
\hline
\end{tabular}

${ }^{\dagger}$ Adjusted for age, sex, glucocorticoids use, and comorbidities. Cl: confidence interval; OR: odds ratio; PPI: proton pump inhibitor. omeprazole, esomeprazole, lansoprazole, pantoprazole, and rabeprazole.

Chi-square test was used to compare baseline characteristics of each categorical variable. Multivariate Cox's regression was used to examine the influence of PPIs on pneumonia in individuals with COPD, and the odds ratios (OR) with 95\% confidence interval (CI) were reported. A two-tailed $\mathrm{P}$ value of $<0.05$ was considered statistically significant. All statistical analyses were performed using SPSS v.18.0 for Windows (SPSS, Inc., Chicago, IL, USA).

Table 3. Odds Ratios and 95\% Confidence Interval of Pneumonia Associated With Risk Factors in Conditional Multivariate Logistic Regression Analysis by Age

\begin{tabular}{ccc}
\hline Drug use & OR $(\mathbf{9 5 \%} \mathbf{C I})$ & OR $^{\dagger} \mathbf{( 9 5 \% ~ C I )}$ \\
\hline Age $<70$ & & \\
None & 1.00 (Reference) & 1.00 (Reference) \\
PPI & $1.07(0.85-1.35)$ & $1.04(0.83-1.31)$ \\
Age $\geq 70$ & & \\
None & 1.00 (Reference) & 1.00 (Reference) \\
PPI & $0.97(0.81-1.15)$ & $0.96(0.81-1.15)$ \\
\hline
\end{tabular}

${ }^{\dagger}$ Adjusted for age, sex, glucocorticoids use, and comorbidities. Cl: confidence interval; OR: odds ratio; PPI: proton pump inhibitor. 
Table 4. Odds Ratios and $95 \%$ Confidence Interval of Pneumonia Associated With Risk Factors in Conditional Multivariate Logistic Regression Analysis by Drug Dosage

\begin{tabular}{lll}
\hline Drug use & OR $(\mathbf{9 5} \%$ CI $)$ & OR $^{\dagger}(\mathbf{9 5} \%$ CI $)$ \\
\hline None & 1.00 (Reference) & 1.00 (Reference) \\
PPI $\leq 30$ days & $1.07(0.51-2.24)$ & $1.12(0.53-2.34)$ \\
PPI $30-90$ days & $0.90(0.76-1.08)$ & $0.86(0.72-1.03)$ \\
PPI $>90$ days & $1.04(0.88-1.24)$ & $0.97(0.81-1.15)$ \\
\hline
\end{tabular}

${ }^{\dagger}$ Adjusted for age, sex, glucocorticoids use, and comorbidities. Cl: confidence interval; OR: odds ratio; PPI: proton pump inhibitor.

\section{Results}

A total of 10,131 patients with COPD were identified, which included 3,377 cases with pneumonia and 6,754 without. Table 1 lists the demographic characteristics, medical conditions, and medication use of each group of patients. The distributions of age and gender between these two groups were not significantly different. There were higher prevalence rates of preexisting diseases in patients with pneumonia than in those without, including CAD (29.9\% vs. $26.9 \%, \mathrm{P}=0.002)$, hypertension (54.1\% vs. $47.9 \%, \mathrm{P}=0.001)$, DM (25.1\% vs. $20.0 \%$, $\mathrm{P}=0.001)$, HF $(11.8 \%$ vs. $8.8 \%, \mathrm{P}=0.001)$ and $\mathrm{CKD}(4.5 \%$ vs. $3.2 \%, P=0.002)$, or concurrent prescriptions of glucocorticoid use $(32.2 \%$ vs. $25 \%, \mathrm{P}=0.001)$.

Among the enrolled patients with pneumonia, $213(5.3 \%)$ cases were found to be using concurrent PPIs. Similarly, there were $436(6.5 \%)$ cases with concurrent PPI use in the without pneumonia subgroup. The rates of concurrent PPI use between with and without pneumonia groups were not significantly different.

The strength of the association between medical history of PPIs and pneumonia is shown in Table 2. After adjustment for measured potential confounders, including concurrent prescriptions of glucocorticoids and comorbidities, the risk of pneumonia was similar whether the patients had used concurrent PPIs or not (adjusted OR $=0.96 ; 95 \%$ CI: $0.83-1.10$ ).

With respect to age, as shown in Table 3 , the prescriptions of PPIs had no effect on pneumonia in the younger patients, defined as younger than 70 years old (adjusted OR $=1.04 ; 95 \%$ CI: 0.83 - 1.10). Similarly, no effect of PPI prescriptions on the risk of pneumonia was seen in elderly cases, defined as 70 years old or older (adjusted OR $=0.96 ; 95 \%$ CI: $0.81-1.15$ ).

Table 4 shows the effect of duration of PPIs prescriptions. There were no significant associations between PPI use and the risk of pneumonia for prescriptions less than 30 days (adjusted $\mathrm{OR}=1.12$, CI: $0.53-2.34), 30-90$ days (adjusted $\mathrm{OR}=0.86$, CI: $0.72-1.03$ ), or longer than 90 days (adjusted $\mathrm{OR}=0.97$, CI: $0.81-1.15)$.

\section{Discussion}

COPD is a chronic, slowly progressive disease characterized by airway obstruction [1]. Individuals with COPD are prone to gastroesophageal reflux disease (GERD), measured using both the GERD-specific questionnaire [9, 10] and 24-h esophageal $\mathrm{pH}$ monitoring $[2,11]$. PPI is the major therapy for GERD based on its effective acid suppressive role.

Previous studies found that prescription of PPIs was associated with an increased risk of pneumonia. For example, three meta-analyses of five to nine observational studies found that PPI use was associated with an increased risk of pneumonia (OR from 1.34 to 1.65 ), especially for a shorter duration of exposure $[4,12,13]$. It has been hypothesized that PPIs induce overgrowth of bacteria in the stomach and subsequently increase the risk for micro-aspiration of bacteria [14]. Furthermore, PPIs also reduce the acidity of the upper aerodigestive tract by activating proton pumps in the larynx and lungs, thus resulting in increased bacterial colonization of these locations and therefore contributing to an increased incidence of pneumonia [15]. However, the basic demographic data of the enrolled cases in these studies were heterogeneous, and the cases with concurrent PPI use and pneumonia might have had worse general health status and a greater degree of immunocompromise.

Furthermore, some studies yielded conflicting results. A retrospective analysis of 31 trials of esomeprazole found a similar rate of pneumonia between those receiving PPI and those treated with placebo or a non-PPI comparator $(\mathrm{OR}=$ 0.94; 99\% CI: 0.29 - 3.07) [16]. A systematic review and metaanalysis of seven randomized controlled trials found that the rate of PPI-associated respiratory tract infections was low (4\% overall; OR: 1.42 ; 95\% CI: $0.86-2.35)$ and the incidence of pneumonia was not specified [17].

In the present study, we investigated patients with COPD, a chronic disease in which pneumonia is a major cause of mortality. Our results found concurrent PPIs had no effect on risk of pneumonia after adjustment for measured potential confounders, including CAD, hypertension, DM, HF, CKD, and glucocorticoid use (adjusted OR $=0.96 ; 95 \%$ CI: $0.83-1.10$ ). PPIs had no impact on risk of pneumonia in younger cases (adjusted OR $=1.04 ; 95 \% \mathrm{CI}: 0.83-1.10$ ) or in elderly cases (adjusted OR $=0.96$; 95\% CI: $0.81-1.15$ ). Similarly, shortterm (less than 30 days; adjusted OR $=1.12$, CI: $0.53-2.34$ ) and long-term (longer than 90 days; adjusted $\mathrm{OR}=0.97, \mathrm{CI}$ : $0.81-1.15$ ) prescriptions of PPIs had no significant association with the risk of pneumonia.

This study had some limitations. First, respiratory illnesses, such as pneumonia and COPD may be misdiagnosed, with one disease being diagnosed as another very similar disease. Absence of corroborating clinical data such as culture specimens or radiographic imaging may result in a false positive or negative classification of pneumonia. Second, over-the-counter medication use was not included in the database used in the study, and data regarding the indication for acid suppression were not available in this study. Third, there were no available data on the patients' lifestyle habits, including smoking status and alcohol consumption, which might be risk factors for pneumonia. Finally, even after adjustment for potential confounders, confounding by indication and disease severity may still be unmatched, as individuals prescribed PPIs are likely to have unobserved health characteristics that predispose them to pneumonia when compared to nonusers. Therefore, further 
research on the relationship between PPIs and pneumonia in patients with COPD is required.

\section{Conclusions}

In this population-based case-control study, PPIs did not contribute to a great occurrence of pneumonia in COPD patients compared with non-users. Further research is required.

\section{References}

1. Pauwels RA, Rabe KF. Burden and clinical features of chronic obstructive pulmonary disease (COPD). Lancet. 2004;364(9434):613-620.

2. Kempainen RR, Savik K, Whelan TP, Dunitz JM, Herrington CS, Billings JL. High prevalence of proximal and distal gastroesophageal reflux disease in advanced COPD. Chest. 2007;131(6):1666-1671.

3. Zielinski J, MacNee W, Wedzicha J, Ambrosino N, Braghiroli A, Dolensky J, Howard P, et al. Causes of death in patients with COPD and chronic respiratory failure. Monaldi Arch Chest Dis. 1997;52(1):43-47.

4. Johnstone J, Nerenberg K, Loeb M. Meta-analysis: proton pump inhibitor use and the risk of community-acquired pneumonia. Aliment Pharmacol Ther. 2010;31(11):11651177.

5. Laheij RJ, Van Ijzendoorn MC, Janssen MJ, Jansen JB. Gastric acid-suppressive therapy and community-acquired respiratory infections. Aliment Pharmacol Ther. 2003;18(8):847-851.

6. Laheij RJ, Sturkenboom MC, Hassing RJ, Dieleman J, Stricker BH, Jansen JB. Risk of community-acquired pneumonia and use of gastric acid-suppressive drugs. JAMA. 2004;292(16):1955-1960.

7. Gulmez SE, Holm A, Frederiksen H, Jensen TG, Pedersen C, Hallas J. Use of proton pump inhibitors and the risk of community-acquired pneumonia: a population-based case- control study. Arch Intern Med. 2007;167(9):950-955.

8. Sarkar M, Hennessy S, Yang YX. Proton-pump inhibitor use and the risk for community-acquired pneumonia. Ann Intern Med. 2008;149(6):391-398.

9. Phulpoto MA, Qayyum S, Rizvi N, Khuhawar SM. Proportion of gastroesophageal reflux symptoms in patients with chronic obstructive pulmonary disease. J Pak Med Assoc. 2005;55(7):276-279.

10. Mokhlesi B, Morris AL, Huang CF, Curcio AJ, Barrett TA, Kamp DW. Increased prevalence of gastroesophageal reflux symptoms in patients with COPD. Chest. 2001;119(4):1043-1048.

11. Casanova C, Baudet JS, del Valle Velasco M, Martin JM, Aguirre-Jaime A, de Torres JP, Celli BR. Increased gastrooesophageal reflux disease in patients with severe COPD. Eur Respir J. 2004;23(6):841-845.

12. Eom CS, Jeon CY, Lim JW, Cho EG, Park SM, Lee KS. Use of acid-suppressive drugs and risk of pneumonia: a systematic review and meta-analysis. CMAJ. 2011;183(3):310319.

13. Giuliano C, Wilhelm SM, Kale-Pradhan PB. Are proton pump inhibitors associated with the development of community-acquired pneumonia? A meta-analysis. Expert Rev Clin Pharmacol. 2012;5(3):337-344.

14. Williams C, McColl KE. Review article: proton pump inhibitors and bacterial overgrowth. Aliment Pharmacol Ther. 2006;23(1):3-10.

15. Altman KW, Waltonen JD, Tarjan G, Radosevich JA, Haines GK, 3rd. Human lung mucous glands manifest evidence of the $\mathrm{H}+/ \mathrm{K}+-\mathrm{ATPase}$ proton pump. Ann Otol Rhinol Laryngol. 2007;116(3):229-234.

16. Estborn L, Joelson S. Occurrence of community-acquired respiratory tract infection in patients receiving esomeprazole: retrospective analysis of adverse events in 31 clinical trials. Drug Saf. 2008;31(7):627-636.

17. Sultan N, Nazareno J, Gregor J. Association between proton pump inhibitors and respiratory infections: a systematic review and meta-analysis of clinical trials. Can J Gastroenterol. 2008;22(9):761-766. 